concentrations of 11 of the host markers and 8 of the markers detected in saliva changed during treatment, indicating that they may be useful in monitoring of TB treatment response.

Conclusions We have identified novel plasma and salivary biosignatures which may be useful in the diagnosis of TB disease and monitoring of the response to TB treatment. Our findings have potential to be translated into point-of-care screening tests after further validation.

\section{PA-064 IDENTIFICATION OF NOVEL PLASMA AND SALIVARY BIOSIGNATURES FOR THE DIAGNOSIS OF TB DISEASE AND MONITORING OF TREATMENT RESPONSE}

Ruschca Jacobs, Novel Chegou, Gerhard Walzl. Stellenbosch University, South Africa

10.1136/bmjgh-2016-000260.97

Background New tools are urgently needed for the rapid diagnosis of TB disease, especially in resource-constrained settings. We investigated the usefulness of host markers detected in plasma and saliva as biomarkers for the diagnosis of TB disease and monitoring of treatment response.

Methods We prospectively collected plasma and saliva samples from 55 individuals that presented with signs and symptoms suggestive of TB disease at a health centre in Cape Town, South Africa, prior to the establishment of a clinical diagnosis. Patients were later classified as having TB disease $(n=22)$ or other respiratory diseases (ORD) $(n=33)$, using a combination of clinical, radiological and laboratory findings. The concentrations of 74 host markers were evaluated in plasma and saliva samples from all study participants using a multiplex cytokine platform. Results Out of the 74 host markers evaluated, 18 markers detected in plasma, and two detected in saliva, showed potential as TB diagnostic candidates, with area under the ROC curve $\geq 0.70$. A six-marker plasma biosignature comprising of NCAM, SAP, IL-1 $\rho$, sCD40L, IL-13 and Apo A-1 diagnosed TB disease with a sensitivity of $100 \%$ (95\% CI: 86.3-100\%) and specificity of $89.3 \%(95 \% \mathrm{CI}, 67.6-97.3 \%)$, whereas a five-marker salivary biosignature comprising of IL-1 $\rho$, IL-23, ECM-1, HCC1 and fibrinogen diagnosed TB disease with a sensitivity of $88.9 \%$ (95\% CI: 76.7-99.9\%) and specificity of $89.7 \%$ (95\% CI: 60.4 $-96.6 \%)$, both regardless of HIV status. The plasma 